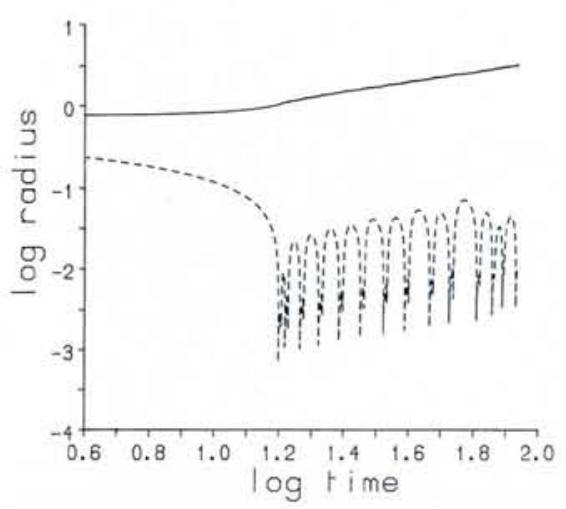

Fig. 5 - Evolution of a star cluster during and after core collapse. The upper curve shows the half-mass radius (the radius of a sphere containing the innermost half of the cluster) plotted against time. In this model the expansion of the cluster is driven by energy emitted by binaries. The lower curve plots the radius of the core (cf. Fig. 2). The collapse is followed by a re-expansion which in this case is unstable to rather regular oscillations. Other models exhibit steady expansion or chaotic oscillations, depending on the size of the cluster, the mechanism of energy generation and other important factors.

born? All these possibilities have their advocates, but they also have their difficulties. Take, for instance, the idea of repeated mergers blowing up as supernovae. It has been shown recently that the presence of all these bright massive stars in a cluster could not have gone unnoticed. On average the core would have to be shining roughly as brightly as all the rest of the cluster put together! No cluster looks like this.

If we think that binary stars are responsible why do we not just look? In recent years there has been a steady growth in evidence, both direct and indirect, for the presence of binary stars in globular star clusters. A few have been discovered directly by careful measurement of variations in the Doppler shifts of their spectra. The indirect evidence includes the discovery, already mentioned, of sources of X-rays in globular star clusters, because these are almost certainly binaries. One star in the binary is a neutron star, and gas from the other star emits X-rays as it falls into the potential well around the neutron star.

Many neutron stars are pulsars, and in the last couple of years a remarkable number of very fast pulsars (so-called millisecond pulsars) have been found in globular star clusters. Just as with the low-mass X-ray sources, their abundance in clusters is much higher than elsewhere in the Galaxy. While some astronomers think that their origin is linked with the X-ray sources, and therefore with binaries, this view is somewhat controversial.

\section{Environmental Issues}

So far we have treated clusters as though they were isolated from the rest of the Universe. But they live inside galaxies, and the stars in them are also attracted by the gravitation of the rest of the galaxy. Up to a point this is just a question of asking how far a star can wander before it escapes from the cluster and becomes an ordinary galactic star. The gravitational effect of the rest of the galaxy, however, varies with time, which complicates the simple picture of an escape distance. When a cluster crosses the plane of the galaxy it is subject to a short-lived, intense gravitational field, the shock of which sets the stars moving more quickly and tends to dissipate the outer parts of the cluster. Much the same thing happens if the cluster comes too close to the nucleus of the galaxy.

It might seem that these processes cause the whole cluster to dissipate, and can combat the tendency of the core to collapse. Certainly they have a big effect on the internal dynamics, but not in the obvious way. If you keep stripping off the outer part of a cluster, the temperature difference between inside and outside is accentuated, and core collapse is actually enhanced. Once again the behaviour of self-gravitating systems is counter-intuitive.

There is one other important process that we have not taken into account. As stars evolve, they lose mass. This is expelled from the cluster, and causes it to expand. In old globular star clusters, this process has ceased to be very important, but when they were young, with lots of massive stars evolving quickly, the effects must have been dramatic. We can still see the evidence in nearby galaxies, especially the Magellanic Clouds, where there are young star clusters as rich as most of the old globular clusters in the galaxy. We can see that their outer parts are being stripped off and will be lost to the rest of the galaxy, leaving a much smaller cluster behind, or perhaps leading to complete disruption.

Indeed the interplay of all these dissipative effects - core evolution, mass loss, external effects - would have led to the complete disruption of clusters if they had not had broadly the properties we see in the globular clusters at the present day. We see clusters of a fairly narrow range of size (masses and radii), not only perhaps because they were born that way, but also because these are the ones that have lasted longest. It is the survival of the fittest on a scale that Darwin could not have imagined.
And so the apparent simplicity of the gravitational $\mathrm{N}$-body problem is very superficial. The evolution of $N$ point masses leads to paradoxical thermodynamic behaviour and even chaotic oscillations. It has revived interest in the three-body problem, because of the importance of binary stars. The dynamics of star clusters is closely linked, through the dynamics of binary stars, with the most exotic stellar-sized objects in the Universe. But for the mathematically minded the dynamics of star clusters has become less tidy. Collisions between the stars play an essential role, and it is becoming clear that the pointmass approximation has had its day.

\section{FURTHER READING}

Binney J. and Tremaine S., Galactic Dynamics (Princeton University Press, Princeton) 1987, chapter 8.

Elson R., Hut P. and Inagaki S., Dynamical Evolution of Globular Clusters, Annual Reviews of Astronomy and Astrophysics, vol. 25, 565-601.

Saslaw W.C., Gravitational Physics of Stellar and Galactic Systems (Cambridge University Press, Cambridge) 1985, chapters 4352.

Spitzer L. Jr., Dynamical Evolution of Globular Clusters (Princeton University Press, Princeton) 1987.

\section{Physical Society of the Soviet Union}

The Physical Society of the Soviet Union, first founded in 1872 and then disbanded in 1930 was to be reinaugurated on 17 November in the Grand Hall of the University of Moscow.

The Society aims to unite scientists and teachers active in fundamental and applied research and in teaching. It will cover education at schools and the universities as well as physics research and development. The intention is to provide qualified and independent expertise on professional issues, expanding and strengthening connexions with other organisations active in the field of science education and technology. Particular support will be given to interdisciplinary research in the field of energy, ecology, economics and global problems.

Reports were circulating a few months ago of the formation of a Moscow Physical Society and even a Leningrad Physical Society that seemed to have been set up as the result of an individualistic initiative, principally to challenge the all-dominant Academy of Sciences. Whether these will continue to function once the USSR Physical Society has been re-instated remains to be seen. 